\title{
A prática da hidroginástica como tratamento complementar para pacientes com transtorno de ansiedade
}

\author{
The practice of hydrogymnastics as a complementary \\ treatment for patients with anxiety disorders \\ José Luiz Lopes Vieira', Mauro Porcu², Viviane Aparecida dos Santos Buzzo
}

\section{RESUMO}

Objetivo: Analisar a efetividade da hidroginástica, como auxiliar terapêutico à redução do nível de ansiedade, em mulheres diagnosticadas com transtorno de ansiedade. Métodos: Este ensaio clínico teve a participação de pacientes com ansiedade, sendo a depressão a comorbidade existente, do programa de Residência Médica em Psiquiatria do Hospital Universitário de Maringá, todas em tratamento com medicamentos ( $n=16$ : grupo experimental = 8; grupocontrole = 8). O experimento foi realizado na piscina aquecida do Departamento de Educação Física da Universidade Estadual de Maringá. O delineamento do estudo foi elaborado com duas sessões de hidroginástica por semana, durante 12 semanas. Os instrumentos utilizados foram: o Inventário de Ansiedade de Beck (BAI) e o Perfil de Estado de Humor (POMS). Para análise estatística, foram utilizados o teste de Friedman, o Teste de Wilcoxon, o Teste de MannWhitney e Comparações Múltiplas, adotando significância em 5\%. Resultados: Os escores do transtorno de ansiedade tiveram redução no grupo experimental, após 12 semanas de intervenção $\left(19,12 \pm 3,12\right.$ para 8,37 $\pm 4,60$ pontos, $\left.P=0,0005^{*}\right)$, e no grupo-controle $(17,87 \pm 14,32$ para 12,12 $\pm 9,58$ pontos, $P=0,254$ ). Para o perfil do estado de humor, o grupo experimental evidenciou perfil de saúde mental positiva, enquanto o grupo-controle demonstrou perfil negativo de estado de humor. Conclusões: Portanto, as pacientes do grupo experimental evidenciaram significativa redução do nível de ansiedade em relação às pacientes do grupocontrole, que utilizaram apenas o tratamento convencional com medicamentos. Para o perfil do estado de humor, foram encontradas alterações no decorrer do estudo; o grupo-controle experimentou alteração negativa de humor durante o ensaio clínico, enquanto os pacientes do grupo experimental evidenciaram perfil positivo de estado de humor com redução da tensão, depressão, raiva, confusão e aumento do vigor.

\section{ABSTRACT}

Objective: To analyze the effectiveness of hydrogymnastics as a therapeutic method to the reduction of anxiety in females diagnosed with anxiety disorder. Methods: This clinical survey had as participants, patients with anxiety, being the depression the existent comorbidade, from Psychia-

1 Universidade Estadual de Maringá/Universidade Estadual de Londrina (UEM/UEL), Departamento de Educação Física.

2 Universidade Estadual de Maringá (UEM), Departamento de Medicina. 


\section{Keywords}

Anxiety disorders, physical exercises, therapeutic complement. try Media Residence Program of the Academical Hospital of Maringá, all had medical treatment ( $n=16$ : experimental group $=8$; control group $=8$ ). The experiment was accomplished in the warm swimming pool of the Department of Physical Education of the State University of Maringá. The design of the study was developed with two hydrogymnastics sessions per week, during 12 weeks. The instruments used in the data collection were: Beck's Anxiety Inventory (BAI) and the Profile of Mood States (POMS). As to statistics analysis the Friedman test, Wilcoxon test, Mann-Whitney test and Multiple Comparisons were used, with a significance of 5\%. Results: Results show that anxiety disorder scores decreased in the experimental group after 12 weeks of intervention (19.12 \pm 3.12 to $\left.8.37 \pm 4.60, P=0.0005^{*}\right)$ and control group $(17.87 \pm 14.32$ to $12.12 \pm 9.58, P=0.254)$. As for mood state profile experimental group evidenced a positive mental health profile, while control group showed a negative mood state profile. Conclusions: It may be concluded that patients from experimental group evidenced significant reduction in anxiety level in relation to control group patients whom used only the conventional medicine treatment. For mood state profile changes were found throughout the study, being that control group experimented negative mood changes during the clinical survey while experimental group patients evidenced positive mood state profile with reduction on tension, depression, anger confusion and enhancement on vigor.

\section{INTRODUÇÃO}

A ansiedade é um estado emocional que inclui componentes psicológicos e fisiológicos, que fazem parte do estado normal das experiências humanas. Mas este estado normal passa a ser patológico quando é desproporcional à situação que o desencadeia, ou quando não existe um objeto específico ao qual o direcione ${ }^{1,2}$.

Situações adversas ou desagradáveis causam um desequilíbrio interno no organismo que culminam com uma resposta biológica e comportamental ao fator estressante 3 . Dessa forma, atribui-se ao estresse uma grande responsabilidade pelo surgimento do transtorno de ansiedade ${ }^{4}$.

Os transtornos de ansiedade estão entre os transtornos psiquiátricos mais frequentes na população geral. Além dos transtornos serem muito frequentes, os sintomas ansiosos estão entre os mais comuns, podendo ser encontrados em qualquer pessoa em determinados períodos de sua existência'. Segundo Kinrys e Wygant (2005)5 , o transtorno de ansiedade possui uma prevalência estimada ao longo da vida de $28,8 \%$ e uma prevalência estimada em 12 meses de $18,1 \%$ na população geral. Atualmente, os profissionais da área médica, que se dedicam às pesquisas sobre a saúde mental, estudam a quantidade, os tipos e os efeitos da ansiedade sobre o organismo e sobre o psiquismo humano, de acordo com as concepções da prática clínica, da medicina psicossomática e da psiquiatria.

Kinrys e Wygant $(2005)^{5}$ relatam que dados de pesquisa americana epidemiológica na população geral dos Estados Unidos demonstraram que mulheres têm mais probabilidade de desenvolver transtorno de ansiedade em comparação com homens. Butler et al. (2005) e Pujol et al. (2002)7 realizaram estudos de neuroimagem e sugerem que o córtex anterior do giro do cíngulo é possivelmente maior e mais ativo entre mulheres com alta resposta ao medo e altos escores de esquiva de dano, em comparação a homens com características semelhantes. Ainda que esses estudos não tenham sido comprovados em pesquisas sobre transtornos de ansiedade específicos e sejam resultados preliminares, podem explicar em parte a maior suscetibilidade de mulheres aos transtornos de ansiedade. Além da neuroimagem, estudos realizados por Redmond $(1997)^{8}$ e Shear (1997) ${ }^{9}$ sugerem que os fatores genéticos e os hormônios sexuais femininos podem desempenhar papéis importantes na expressão dessas diferenças de gênero.

Altchiler e Motta (1994) ${ }^{10}$, Broman-Fulks et al. (2004) ${ }^{11}$, Brown et al. (1993)'12, O'Connor et al. (1995)'13, Petruzzello et al. (1991) ${ }^{14}$ e Raglin et al. (1993) demonstraram que o exercício físico pode ser um auxiliar terapêutico no tratamento da ansiedade. Antunes et al. (2005) ${ }^{16}$ e Melo et al. (2005) ${ }^{17}$ concluíram que um programa de exercício físico aeróbico regular é suficiente para promover modificações favoráveis nos escores indicativos de depressão e ansiedade e melhorar a qualidade de vida. Dessa forma, o objetivo desse estudo foi analisar a efetividade de um programa de exercícios físicos baseado em hidroginástica, como auxiliar terapêutico à redução do nível de ansiedade, em mulheres diagnosticadas com transtorno de ansiedade.

\section{MÉTODOS}

Para realização deste ensaio clínico, foram selecionadas pacientes do programa de Residência Médica em Psiquiatria do Hospital Universitário de Maringá. O experimento foi realizado na piscina aquecida do Departamento de Educa- 
ção Física, pertencente ao Centro de Ciências e da Saúde da Universidade Estadual de Maringá. Participaram do experimento $(n=16)$ mulheres com idade média de $36,45( \pm 9,45)$ anos, com diagnóstico de ansiedade, sendo a depressão a comorbidade existente, de acordo com o diagnóstico realizado pelos residentes da Residência Médica em Psiquiatria, com base nos critérios do DSM-IV.

Assim sendo, para verificação do nível de ansiedade, utilizou-se a aplicação do Inventário de Ansiedade de Beck - BAI (Beck et al.18, 1988 versão traduzida por Cunha, 2001) ${ }^{19}$, que foi aplicado pelos residentes do programa de Residência Médica em Psiquiatria.

Essa escala apresenta 21 itens relacionados a sintomas ansiosos, cada um composto de quatro afirmações que evoluem em um grau de intensidade de 0 a 3. Mais de uma afirmação pode ser escolhida, porém o escore computado é sempre o de maior intensidade. A soma dos escores obtidos em cada item resulta em um escore total, que varia de $0 \mathrm{a} 63$, sendo que acima de 10 pontos equivale à ansiedade grave, escore de 6 a 9 pontos denota a ansiedade modera$\mathrm{da}$, escore de 3 a 5 pontos apresenta ansiedade leve e escore abaixo de 3 pontos significa ausência de ansiedade.

O delineamento do estudo foi realizado com a subdivisão aleatória por sorteio das pacientes em dois grupos (grupo experimental $=8$ e grupo-controle $=8$ ). As pacientes foram avaliadas em três momentos do ensaio clínico: no início do programa, ao final de 6 semanas e ao encerramento do programa de exercícios físicos (12 semanas).

Foram considerados como critério para a seleção da amostra: o quadro clínico de ansiedade, a obrigatoriedade do tratamento convencional com medicamentos para todas as pacientes, o ciclo reprodutivo ativo, a não vinculação a nenhum programa de exercícios físicos regulares, a ausência de outras patologias ou a administração medicamentosa a qual o exercício físico não fosse recomendado e o tipo de psicofármaco utilizado no tratamento da ansiedade (inibidores seletivos de recaptação de serotonina [ISRSs], benzodiazepínicos [BZD] e antidepressivos tricíclicos [ADT]) como se especifica na tabela 1.

Tabela 1. Tipo de medicamento utilizado pelas pacientes que participaram do ensaio clínico, especificando o tipo de psicofármaco utilizado

\begin{tabular}{|c|c|c|}
\hline \multicolumn{3}{|c|}{ Tipos de medicamentos } \\
\hline $\begin{array}{l}\text { Inibidores selettivos de } \\
\text { recaptação de serotonina - ISRS }\end{array}$ & Benzodiazepínicos - BZD & Antidepressivos tricíclicos - ADT \\
\hline $\mathrm{N}=11(\mathrm{GE}=4 ; \mathrm{GC}=7)$ & $\mathrm{n}=2(\mathrm{GE}=1 ; \mathrm{GC}=1)$ & $n=5(G E=4 ; G C=1)$ \\
\hline Citalopram $20 \mathrm{mg}$ & ${ }^{\circ}$ Alprazolam 0,05 mg & Amitriplina $20 \mathrm{mg}$ \\
\hline Fluoxetina $20 \mathrm{mg}$ & 'Clonazepam 0,05 mg & Clomipramina $10 \mathrm{mg}$ \\
\hline Paroxetina $10 \mathrm{mg}$ & & Clomipramina $20 \mathrm{mg}$ \\
\hline aParoxetina $20 \mathrm{mg}$ & & ${ }^{\circ}$ Clomipramina 75 mg \\
\hline & & Imipramina $20 \mathrm{mg}$ \\
\hline
\end{tabular}

Os dados em destaque referem-se à utilização de dois tipos de medicamentos por uma paciente do GE (com o "0" sobrescrito) e por uma paciente do GC (com o "a" sobrescrito). GE: grupo experimental, GC: grupo-controle.
Ainda, como instrumento de medida, foi utilizado o Questionário Internacional de Atividade Física - Versão Curta (IPAQ) ${ }^{20}$, que foi aplicado pela pesquisadora do estudo, nos três momentos do estudo (no início do programa, ao final de 6 semanas e ao encerramento do programa de intervenção) para avaliar o estilo de vida sedentário, assegurando que as pacientes não iriam alterar sua rotina sedentária.

Esse ensaio clínico foi submetido à aprovação do Comitê de Ética em Pesquisa envolvendo Seres Humanos da Universidade Estadual de Maringá (Protoc.363/2006) e à autorização da Superintendência do Hospital Universitário de Maringá. Todas as pacientes concordaram em participar do estudo e assinaram um termo de consentimento livre e esclarecido.

Para as pacientes selecionadas, a quantidade/dia administrada do medicamento durante os três meses de pesquisa não foi modificada. O tratamento medicamentoso proporcionado às pacientes do programa de Residência Médica em Psiquiatria foi convencional, por administração do medicamento, para o grupo experimental, e o complemento terapêutico utilizado, além do medicamento, foi um programa de exercícios físicos, baseado em hidroginástica.

O grupo experimental foi submetido a um programa de exercícios físicos baseado em hidroginástica, com frequência de duas sessões semanais, cada uma com duração de 50 minutos. Esse programa de exercícios físicos foi ministrado por uma professora de Educação Física especializada em hidroginástica. A intensidade das sessões de exercícios físicos foi mensurada por meio da Escala de Percepção Subjetiva do Esforço21, e a pressão arterial foi aferida antes e após o exercício físico, no grupo experimental e quinzenalmente no grupo-controle. Os resultados encontrados durante o programa de hidroginástica para a percepção subjetiva do esforço e para a pressão arterial não apresentaram alterações negativas, demonstrando, deste modo, a efetividade do programa de hidroginástica quanto sua segurança. Além do controle dessas variáveis físicas durante o exercício físico, utilizou-se também um protocolo para avaliar o estado de humor das pacientes (Perfil do Estado de Humor - POMS)22. Esse protocolo verifica os níveis de tensão, depressão, raiva, vigor, fadiga e confusão, relacionando-se ao humor do indivíduo de acordo com a esfera estressante do ambiente em que está inserido. A escala enumera um grande número de sentimentos, em que o sujeito afere valores de 0 , quando sente que não há manifestação, a 4, quando se acredita extremamente acometido pelo sentimento. Para analisar seus resultados representativos em uma tendência emocional, os sentimentos foram agrupados posteriormente na sua classificação de humor, e a cada um foi submetido um valor que soma ou subtrai, traçando dessa forma o perfil do estado de humor do indivíduo naquela dada circunstância. Essa medida foi realizada para que fosse possível controlar as modificações psicológicas ao decorrer do programa. Esse protocolo foi aplicado uma vez por semana, antes e após o exercício físico, nas pacientes do grupo experimental e, quinzenalmente, durante 
as 12 semanas, nas pacientes do grupo-controle. Todas as medidas (exceto o Inventário de Ansiedade de Beck) foram coletadas pela pesquisadora do estudo.

Na análise estatística, verificou-se a normalidade dos dados por meio do Teste de Kolmogorov-Smirnov, sendo utilizados o Teste de Friedman para comparações intra-grupos dependentes, o Teste de Wilcoxon para comparações intragrupos dependentes, o Teste de Mann-Whitney para comparações entre grupos independentes e comparações múltiplas para verificar possíveis diferenças intra e entre grupos nos três momentos do estudo (pré-teste, teste intermediário e pós-teste), adotando-se $p<0,05$.

\section{RESULTADOS}

Inicialmente, apresentam-se os resultados referentes aos efeitos do programa de exercícios físicos regulares sobre os níveis de ansiedade do grupo submetido ao programa de intervenção e entre os grupos do estudo. Posteriormente, serão apresentados os resultados do perfil de estado de humor do grupo praticante de hidroginástica, pré e pós-sessão de exercício físico, e os resultados do grupo que não fez uso do exercício físico como tratamento complementar na linha de base, 6a semana e $12^{a}$ semana de intervenção e as comparações entre eles.

Com relação aos escores de ansiedade entre as pacientes do grupo experimental e as pacientes do grupo-controle, os resultados encontrados não apresentaram diferenças estatisticamente significativas na linha de base, demonstrando a homogeneidade dos grupos. Na comparação intragrupos verifica-se diferença estatisticamente significativa apenas para as pacientes submetidas ao programa de intervenção (grupo experimental). Dessa forma, os escores médios da ansiedade (Tabela 2) demonstram que o grupo experimental apresentou redução estatisticamente significativa que se deu da linha de base para a $12^{\text {a }}$ semana de intervenção. No grupo-controle ocorreu redução do nível de ansiedade nas 6 primeiras semanas e permanecendo estável até a $12^{a}$ semana.

Tabela 2. Média e desvio-padrão da redução do nível de ansiedade de pacientes praticantes e não praticantes de um programa de exercícios físicos baseado em hidroginástica

\begin{tabular}{|c|c|c|c|c|c|c|}
\hline \multicolumn{7}{|c|}{ Inventário de ansiedade de Beck - BAI } \\
\hline & & la de base & & semana & $12^{\mathrm{a}}$ semana & Valordep \\
\hline $\begin{array}{l}\text { Grupo } \\
\text { experimental }\end{array}$ & 19,12 & $\pm 8,47$ & 12,62 & $\pm 6,41$ & $8,37 \pm 4,60$ & $0,0005^{*}$ \\
\hline Grupo-controle & 17,87 & $\pm 14,32$ & 12,00 & $\pm 9,08$ & $12,12 \pm 9,58$ & 0,2540 \\
\hline
\end{tabular}

${ }^{*} p<0,05$ entre a linha de base e a 12 semana do programa de intervenção.

Os escores médios do perfil de estado de humor (POMS) na linha de base, na $6^{\text {a }}$ semana e na $12^{\text {a }}$ semana, para o grupo praticante de exercício físico (grupo experimental) e não praticante de exercício físico (grupo-controle), estão representados nas tabelas 3, 4, 5 e 6 .
Tabela 3. Média e desvio-padrão para o perfil de estado de humor de pacientes praticantes (GE) e não praticantes (GC) de um programa de exercício físico baseado em hidroginástica

\begin{tabular}{|c|c|c|c|c|c|c|c|}
\hline \multicolumn{8}{|c|}{ POMS } \\
\hline & \multicolumn{2}{|c|}{ Linha de base } & \multicolumn{2}{|c|}{ 6a semana } & \multicolumn{2}{|c|}{$12^{\mathrm{a}}$ semana } & \multirow{2}{*}{ Valor de } \\
\hline GE-Pré & M & SD & M & SD & $M$ & SD & \\
\hline Tensão & 4,52 & 3,08 & 4,15 & 3,41 & 5,01 & 5,08 & 0,875 \\
\hline Depressão & 3,87 & 6,10 & 2,37 & 4,60 & 2,62 & 5,04 & 0,946 \\
\hline Raiva & 1,75 & 4,20 & 1,50 & 2,20 & 2,00 & 4,28 & 0,291 \\
\hline Vigor & 13,62 & 4,66 & 13,37 & 2,13 & 14,87 & 4,01 & 0,261 \\
\hline Fadiga & 3,87 & 1,12 & 2,37 & 3,20 & 3,12 & 3,60 & 0,497 \\
\hline Confusão & 2,62 & 3,20 & 2,87 & 2,75 & 1,87 & 2,17 & 0,260 \\
\hline \multicolumn{8}{|l|}{ GE-Pós } \\
\hline Tensão & 3,12 & 3,08 & 2,26 & 3,41 & 3,00 & 5,08 & 0,325 \\
\hline Depressão & 1,12 & 6,10 & 1,00 & 4,60 & 0,75 & 5,04 & 0,401 \\
\hline Raiva & 0,50 & 4,20 & 0,87 & 2,20 & 0,87 & 4,28 & 0,735 \\
\hline Vigor & 18,00 & 4,66 & 15,75 & 2,13 & 17,50 & 4,01 & 0,883 \\
\hline Fadiga & 1,87 & 1,12 & 2,62 & 3,20 & 3,75 & 3,60 & 0,786 \\
\hline Confusão & 1,50 & 3,20 & 1,37 & 2,75 & 2,50 & 2,17 & 0,469 \\
\hline \multicolumn{8}{|l|}{ GC } \\
\hline Tensão & 3,60 & 4,45 & 7,59 & 7,15 & 7,69 & 8,34 & 0,140 \\
\hline Depressão & 4,87 & 5,14 & 7,12 & 5,38 & 9,00 & 8,36 & 0,174 \\
\hline Raiva & 5,87 & 6,66 & 4,75 & 2,91 & 7,37 & 6,18 & 0,417 \\
\hline Vigor & 11,12 & 4,15 & 10,62 & 4,98 & 9,50 & 6,37 & 0,531 \\
\hline Fadiga & 5,37 & 4,98 & 8,25 & 6,18 & 7,00 & 4,78 & 0,798 \\
\hline Confusão & 4,25 & 4,83 & 4,75 & 3,65 & 7,37 & 5,95 & 0,197 \\
\hline
\end{tabular}

* $p<0,05$.

GE-pré: grupo experimental pré-sessão de hidroginástica, GE-pós: grupo experimental pós-sessão de hidroginástica, GC: grupo-controle.

Tabela 4. Comparação do perfil de estado de humor entre pré e pós-sessão de hidroginástica do grupo submetido ao programa de exercícios físicos (GE)

\begin{tabular}{|c|c|c|c|c|c|c|}
\hline \multirow[t]{2}{*}{ Teste POMS } & \multirow[t]{2}{*}{ Escore } & \multicolumn{2}{|c|}{ GE-Pré } & \multicolumn{2}{|c|}{ GE-Pós } & \multirow[t]{2}{*}{ Valor de $p$} \\
\hline & & M & SD & M & SD & \\
\hline \multirow[t]{3}{*}{ Tensão } & Linha base & 4,52 & 3,08 & 3,12 & 3,18 & 0,121 \\
\hline & $6^{a}$ semana & 4,15 & 3,41 & 2,26 & 2,11 & 0,113 \\
\hline & $12^{\mathrm{a}}$ semana & 5,01 & 5,08 & 3,00 & 2,00 & 0,418 \\
\hline \multirow[t]{3}{*}{ Depressão } & Linha base & 3,87 & 6,10 & 1,12 & 1,12 & 0,247 \\
\hline & 6a semana & 2,37 & 4,60 & 1,00 & 1,93 & 0,371 \\
\hline & $12^{\mathrm{a}}$ semana & 2,62 & 5,04 & 0,75 & 1,39 & 0,181 \\
\hline \multirow[t]{3}{*}{ Raiva } & Linha base & 1,75 & 4,20 & 0,50 & 1,41 & 0,371 \\
\hline & $6^{\mathrm{a}}$ semana & 1,50 & 2,20 & 0,87 & 1,64 & 0,089 \\
\hline & $12^{\mathrm{a}}$ semana & 2,00 & 4,28 & 0,87 & 1,64 & 0,371 \\
\hline \multirow[t]{3}{*}{ Vigor } & Linha base & 13,37 & 4,66 & 18,00 & 6,30 & $0,024^{*}$ \\
\hline & $6^{a}$ semana & 13,37 & 2,13 & 15,75 & 3,45 & 0,171 \\
\hline & $12^{\mathrm{a}}$ semana & 14,87 & 4,01 & 17,50 & 4,60 & 0,270 \\
\hline \multirow[t]{3}{*}{ Fadiga } & Linha base & 3,87 & 1,12 & 1,87 & 1,55 & $0,033^{*}$ \\
\hline & $6^{a}$ semana & 2,37 & 3,20 & 2,62 & 2,39 & 0,931 \\
\hline & $12^{\mathrm{a}}$ semana & 3,12 & 3,60 & 3,75 & 3,10 & 0,496 \\
\hline \multirow[t]{3}{*}{ Confusão } & Linha base & 2,62 & 3,20 & 1,50 & 2,33 & 0,276 \\
\hline & $6^{a}$ semana & 2,87 & 2,75 & 1,37 & 1,99 & 0,057 \\
\hline & $12^{\mathrm{a}}$ semana & 1,87 & 2,17 & 2,50 & 2,20 & 0,167 \\
\hline
\end{tabular}

${ }^{*} p<0,05$.

GE-Pré: grupo experimental pré-sessão de hidroginástica, GE-Pós: grupo experimental pós-sessão de hidroginástica. 
Tabela 5. Comparação do perfil de estado de humor entre grupo submetido ao programa de exercícios físicos (GE), pré-sessão de hidroginástica, e o grupo não praticante de exercícios físicos (GC)

\begin{tabular}{|c|c|c|c|c|c|c|}
\hline \multirow[t]{2}{*}{ Teste POMS } & \multirow[t]{2}{*}{ Escore } & \multicolumn{2}{|c|}{ GE-Pré } & \multicolumn{2}{|c|}{ GC } & \multirow[t]{2}{*}{ Valor dep } \\
\hline & & M & SD & M & SD & \\
\hline \multirow[t]{3}{*}{ Tensão } & Linha base & 4,52 & 3,08 & 3,60 & 4,45 & 0,366 \\
\hline & $6^{\mathrm{a}}$ semana & 4,15 & 3,41 & 7,59 & 7,15 & 0,491 \\
\hline & $12^{a}$ semana & 5,01 & 5,08 & 7,69 & 8,34 & 0,395 \\
\hline \multirow[t]{3}{*}{ Depressão } & Linha base & 3,87 & 6,10 & 4,87 & 5,14 & 0,660 \\
\hline & $6^{\mathrm{a}}$ semana & 2,37 & 4,60 & 7,12 & 5,38 & $0,035^{*}$ \\
\hline & $12^{\mathrm{a}}$ semana & 2,62 & 5,04 & 9,00 & 8,36 & $0,046^{*}$ \\
\hline \multirow[t]{3}{*}{ Raiva } & Linha base & 1,75 & 4,20 & 5,87 & 6,66 & 0,072 \\
\hline & $6^{a}$ semana & 1,50 & 2,20 & 4,75 & 2,91 & $0,031^{*}$ \\
\hline & $12^{\mathrm{a}}$ semana & 2,00 & 4,28 & 7,37 & 6,18 & $0,021^{*}$ \\
\hline \multirow[t]{3}{*}{ Vigor } & Linha base & 13,62 & 4,66 & 11,12 & 4,15 & 0,342 \\
\hline & $6^{\mathrm{a}}$ semana & 13,37 & 2,13 & 10,62 & 4,98 & 0,459 \\
\hline & $12^{\mathrm{a}}$ semana & 14,87 & 4,01 & 9,50 & 6,37 & 0,058 \\
\hline \multirow[t]{3}{*}{ Fadiga } & Linha base & 3,87 & 1,12 & 5,37 & 4,98 & 0,665 \\
\hline & $6^{a}$ semana & 2,37 & 3,20 & 8,25 & 6,18 & $0,019^{*}$ \\
\hline & $12^{\mathrm{a}}$ semana & 3,12 & 3,60 & 7,00 & 4,78 & 0,089 \\
\hline \multirow[t]{3}{*}{ Confusão } & Linha base & 2,62 & 3,20 & 4,25 & 4,83 & 0,487 \\
\hline & $6^{a}$ semana & 2,87 & 2,75 & 4,75 & 3,65 & 0,424 \\
\hline & $12^{\mathrm{a}}$ semana & 1,87 & 2,17 & 7,37 & 5,95 & $0,017^{*}$ \\
\hline
\end{tabular}

${ }^{*} p<0,05$.

GE-Pré: grupo experimental pré-sessão de hidroginástica, GC: grupo-controle.

Tabela 6. Comparação do perfil de estado de humor entre o grupo submetido ao programa de exercícios físicos (GE), pós-sessão de hidroginástica, e o grupo não praticante de exercícios físicos(GC)

\begin{tabular}{|c|c|c|c|c|c|c|}
\hline \multirow[t]{2}{*}{ Teste POMS } & \multirow[t]{2}{*}{ Escore } & \multicolumn{2}{|c|}{ GE-Pós } & \multicolumn{2}{|c|}{ GC } & \multirow[t]{2}{*}{ Valor de $p$} \\
\hline & & M & SD & M & SD & \\
\hline \multirow[t]{3}{*}{ Tensão } & Linha base & 3,12 & 3,08 & 3,60 & 4,45 & 0,914 \\
\hline & $6^{\mathrm{a}}$ semana & 2,26 & 3,41 & 7,59 & 7,15 & 0,056 \\
\hline & $12^{\mathrm{a}}$ semana & 3,00 & 5,08 & 7,69 & 8,34 & 0,137 \\
\hline \multirow[t]{3}{*}{ Depressão } & Linha base & 1,12 & 6,10 & 4,87 & 5,14 & 0,194 \\
\hline & $6^{\mathrm{a}}$ semana & 1,00 & 4,60 & 7,12 & 5,38 & $0,002^{*}$ \\
\hline & $12^{\mathrm{a}}$ semana & 0,75 & 5,04 & 9,00 & 8,36 & $0,008^{*}$ \\
\hline \multirow[t]{3}{*}{ Raiva } & Linha base & 0,50 & 4,20 & 5,87 & 6,66 & $0,010^{*}$ \\
\hline & 6a semana & 0,87 & 2,20 & 4,75 & 2,91 & $0,009^{*}$ \\
\hline & $12^{\mathrm{a}}$ semana & 0,87 & 4,28 & 7,37 & 6,18 & $0,009^{*}$ \\
\hline \multirow[t]{3}{*}{ Vigor } & Linha base & 18,00 & 4,66 & 11,12 & 4,15 & $0,049^{*}$ \\
\hline & $6^{\mathrm{a}}$ semana & 15,75 & 2,13 & 10,62 & 4,98 & 0,101 \\
\hline & $12^{\mathrm{a}}$ semana & 17,50 & 4,01 & 9,50 & 6,37 & $0,026^{*}$ \\
\hline \multirow[t]{3}{*}{ Fadiga } & Linha base & 1,87 & 1,12 & 5,37 & 4,98 & 0,120 \\
\hline & $6^{a}$ semana & 2,62 & 3,20 & 8,25 & 6,18 & $0,022^{*}$ \\
\hline & $12^{\mathrm{a}}$ semana & 3,75 & 3,60 & 7,00 & 4,78 & 0,125 \\
\hline \multirow[t]{3}{*}{ Confusão } & Linha base & 1,50 & 3,20 & 4,25 & 4,83 & 0,152 \\
\hline & $6^{\mathrm{a}}$ semana & 1,37 & 2,75 & 4,75 & 3,65 & $0,025^{*}$ \\
\hline & $12^{\mathrm{a}}$ semana & 2,50 & 2,17 & 7,37 & 5,95 & $0,033^{*}$ \\
\hline
\end{tabular}

${ }^{*} p<0,05$

GE-Pós: grupo experimental pós-sessão de hidroginástica, GC: grupo-controle.
O grupo submetido ao programa de hidroginástica (grupo experimental) na pré e na pós-sessão de exercício aferido pelo POMS na linha de base, $6^{\text {a }}$ semana e $12^{\text {a }}$ semana não demonstrou diferença estatisticamente significativa, porém ambos apresentaram alteração positiva, ou seja, as médias para o sentimentos "negativos" diminuíram e a média para o sentimento "positivo" aumentou, no decorrer do programa de intervenção, corroborando, desse modo, alteração positiva do humor. Já para o grupo não praticante de exercícios físicos (grupo-controle), além de não apresentar diferença estatisticamente significativa, as médias demonstraram alteração negativa dos sentimentos, pois as emoções de tensão, depressão, raiva e confusão, com exceção da fadiga, aumentaram e o sentimento "positivo" vigor diminuiu no decorrer do experimento, evidenciando alteração negativa do humor (Tabela 3).

As médias pré e pós-sessão do exercício físico para o perfil de estado de humor no grupo submetido ao programa de hidroginástica (grupo experimental) demonstraram diferença estatisticamente significativa apenas para os sentimentos vigor e fadiga, ambos na linha de base. Para o sentimento vigor, as pacientes demonstraram aumento do sentimento, no entanto, para a fadiga ocorreu o inverso, pois houve uma diminuição do sentimento da pré-sessão para a pós-sessão de exercício físico. Para os sentimentos tensão, depressão, raiva e confusão ocorreram alterações consideradas positivas, pois houve diminuição em ambos os sentimentos, no entanto, não foram apresentadas diferenças estatisticamente significativas (Tabela 4).

Quando realizada a comparação dos escores do perfil de estado de humor entre a pré-sessão de exercício físico do grupo submetido ao programa de hidroginástica (grupo experimental pré-sessão) e o grupo não praticante de exercícios físicos (grupo-controle), verificou-se diferença estatisticamente significativa nos sentimentos "negativos" depressão, raiva, fadiga e confusão, como demonstra a tabela 5. Para o sentimento depressão, a média apresentada para o grupo-controle foi superior ao grupo experimental na pré-sessão, na $6^{a}$ semana e na $12^{2}$ semana, evidenciando, desse modo, melhora do quadro depressivo das pacientes do grupo experimental (pré-sessão de hidroginástica) no decorrer do estudo. Para a raiva, a média na $6^{\mathrm{a}}$ e na $12^{\mathrm{a}}$ semana também foi superior. O mesmo ocorreu com o sentimento fadiga na $6^{a}$ semana e para o sentimento confusão na $12^{\text {a }}$ semana do experimento, evidenciando, do mesmo modo, diminuição dos sentimentos negativos para o grupo submetido ao programa de hidroginástica (grupo experimental na pré-sessão) (Tabela 5).

Na comparação dos escores do perfil de estado de humor entre a pós-sessão de exercício físico do grupo submetido ao programa de hidroginástica (grupo experimental) e o grupo não praticante de exercícios físicos (grupo-controle), constatou-se diferença estatisticamente significativa nos sentimentos depressão, raiva, vigor, fadiga e confusão como 
demonstra a tabela 6. Nos sentimentos "negativos" depressão, raiva, fadiga e confusão as médias foram superiores para o grupo-controle, quando comparado com o grupo experimental na pós-sessão de hidroginástica, enquanto para o sentimento "positivo" vigor a média foi inferior para o grupocontrole, demonstrando, assim, diminuição dos sentimentos "negativos" e aumento do sentimento "positivo" para o grupo submetido ao programa de hidroginástica (grupo experimental pós-sessão). Para depressão, encontrou-se diferença estatisticamente significativa na $6^{\mathrm{a}}$ e $12^{\mathrm{a}}$ semana de intervenção, e para raiva evidenciaram-se diferenças na linha de base, $6^{a}$ semana e $12^{a}$ semana. Nos dois últimos sentimentos "negativos" fadiga e confusão, as diferenças foram encontradas na $6^{a}$ semana e, para confusão, na $12^{a}$ semana de intervenção. Por último, para o sentimento "positivo" vigor averiguou-se diferença estatisticamente significativa na linha de base e na 12a semana do experimento (Tabela 6).

\section{DISCUSSÃO}

No grupo que participou do programa de exercícios físicos regulares (grupo experimental), a redução da média no préteste de $19,12( \pm 3,12)$ para 8,37 $( \pm 4,59)$ pontos após 12 semanas demonstrou evidências na efetividade dos exercícios físicos regulares do nível de ansiedade. E o grupo que não participou do programa de intervenção, baseado em hidroginástica (grupo-controle), também apresentou redução da média no pré-teste de 17,87 ( \pm 14,31) para 12,12 ( \pm 9,58) pontos após 12 semanas, evidenciando também, apesar de não terem sido encontrados resultados estatisticamente significativos, a efetividade do medicamento no tratamento do transtorno de ansiedade. Portanto, verifica-se que o tratamento medicamentoso quando associado ao exercício físico proporciona benefícios superiores ao paciente ansioso quando comparado ao medicamento isolado.

Os resultados encontrados corroboram com Salmon (2001)23, no qual a prática regular de exercícios físicos aeróbios pode produzir efeitos antidepressivos e ansiolíticos e proteger o organismo dos efeitos prejudiciais do estresse na saúde física e mental. Craft e Landers $(1998)^{24}$ e Petruzzello et al. (1991) ${ }^{14}$ relatam que o exercício físico como tratamento para ansiedade e depressão é tão eficaz quanto tratamentos psicoterápicos e farmacológicos, possuindo vantagem de ser mais saudável, econômico e ter maior adesão quanto comparado a esses tratamentos. Brown et al. (1993) ${ }^{12}$ avaliaram os efeitos do exercício físico aeróbio nos níveis de ansiedade de indivíduos com transtorno de ansiedade e concluíram que o exercício físico aeróbio produziu redução da ansiedade com resultados similares às estratégias de meditação e relaxamento.

Gallo Jr. et al. (1995)25, Goldfarb e Jamurtas $(1997)^{26} \mathrm{e}$ Heitkamp et al. (1996) ${ }^{27}$ também defendem a utilização de exercício físico como tratamento complementar do transtorno de ansiedade, quando relatam em seus estudos que durante a realização do exercício físico ocorre liberação de $\beta$-endorfina e do neurotransmissor dopamina pelo organismo, propiciando um efeito tranquilizante e analgésico no praticante regular, que frequentemente se beneficia de um efeito relaxante pós-esforço e, em geral, consegue manter um estado de equilíbrio psicossocial mais estável diante das ameaças do meio externo. Além disso, o exercício físico leva o indivíduo a uma maior participação social, resultando em um bom nível de bem-estar biopsicofísico, fatores esses que contribuem para a melhoria de sua qualidade de vida ${ }^{28-30}$.

Desse modo, o exercício físico pode ser proposto como uma alternativa de prevenção e redução de níveis de ansiedade e melhorar o estado do humor desses pacientes. Baldwin e Polkinghorn (2005) ${ }^{31}$ relatam que, além do exercício físico regular, as medicações também são eficientes no tratamento de pacientes com transtorno de ansiedade, sendo aprovadas por psiquiatras acadêmicos, justificando, assim, a diminuição dos níveis de ansiedade do grupo que não fez uso do exercício físico como tratamento complementar (grupo-controle).

Eckerson e Anderson (1992) ${ }^{32}$, Kruel (2000)33 e Vieira et al. $(2007)^{34}$ relatam que o tipo de atividade e o ambiente em que o exercício físico está sendo realizado são de extrema importância, pois o indivíduo precisa sentir-se confortável com o tipo de exercício praticado. Dessa forma, o exercício físico em imersão, a hidroginástica, está sendo cada vez mais indicado devido aos seus múltiplos benefícios à saúde, promovendo melhoria nos diversos componentes da aptidão física, como força, flexibilidade, composição corporal e condicionamento cardiorrespiratório ${ }^{35-38}$. Assim, exercícios realizados no meio aquático apresentam um reduzido impacto comparado com o meio terrestre, proporcionando também um ambiente descontraído e atrativo $0^{33,39}$ e um comportamento de frequência cardíaca e pressão arterial mais baixos ${ }^{40,41}$. Esses benefícios que o meio aquático proporciona nos condicionamentos gerais do corpo estão relacionados com as propriedades da água, como: a temperatura adequada da água, em torno de $17^{\circ} \mathrm{C}$ a $34^{\circ} \mathrm{C}$, a resistência da água proporcionando sobrecarga para o trabalho muscular, melhoria do condicionamento cardiovascular e do dispêndio energético. Além disso, a pressão hidrostática auxilia na correção postural, na reeducação respiratória, no conhecimento corporal, no equilíbrio e no retorno venoso ${ }^{40,42}$.

Os efeitos fisiológicos relacionados ao exercício físico podem ser classificados em agudos imediatos, agudos tardios e crônicos. Os efeitos agudos ocorrem em associação direta com a sessão de exercício; os efeitos agudos imediatos ocorrem nos períodos pré e pós-imediato do exercício físico, como elevação da frequência cardíaca, ventilação pulmonar e sudorese; já os efeitos agudos tardios acontecem ao longo das primeiras 24 ou 28 horas, às vezes até 72 horas, 
que se seguem a uma sessão de exercício físico e podem ser identificados na discreta redução dos níveis tensionais. Por último, os efeitos crônicos, também denominados adaptações, resultam da exposição regular às sessões de exercícios físicos e representam aspectos morfofuncionais que diferenciam um indivíduo fisicamente treinando de um indivíduo sedentário ${ }^{43,44}$. O exercício também é capaz de promover a angiogênese, aumentando o fluxo sanguíneo para os músculos esqueléticos e para o músculo cardíaco ${ }^{45,46}$. Isso explica a melhoria da efetividade do programa de hidroginástica entre a $6^{\mathrm{a}} \mathrm{e}$ a $12^{\mathrm{a}}$ semana de intervenção.

Os escores médios para o perfil de estado de humor demonstraram que no grupo de praticantes de exercícios físicos o humor apresentou alterações durante o programa de intervenção, baseado em hidroginástica, ou seja, os sentimentos negativos de tensão, depressão, raiva, fadiga e confusão diminuíram e o sentimento positivo vigor aumentou no decorrer do programa de exercícios físicos. Desse modo, verificou-se presença de alterações positivas no humor, pois os resultados evidenciaram um perfil conhecido como perfil de saúde mental positiva, em que a dimensão vigor se apresenta mais evidente que as outras dimensões ${ }^{47,48}$. Os resultados obtidos neste ensaio clínico para o grupo de praticantes de exercícios físicos são confirmados por Berger $(1996)^{49}$, Berger e Mcinman (1993) $)^{50}$ e Boutcher (1993) ${ }^{51}$, quando afirmam que o exercício físico proporciona benefícios em parâmetros emocionais, prevenindo alterações de humor. O exercício físico ainda está associado com a oportunidade de esquecer os problemas cotidianos por um período de tempo; sentir-se em controle da experiência; sentir-se competente e eficaz; aumentar as interações sociais; fortalecer o autoconceito, a autoestima e o bom humor; diminuir a ansiedade, a hostilidade e a agressividade ${ }^{52-57}$.

Biddle $(1995)^{58}$, Petruzzello et al. (1991) $)^{14}$, Raglin $(1990)^{59} \mathrm{e}$ Raglin e Morgan (1987) ${ }^{60}$ relatam que os efeitos agudos da atividade física proporcionam melhora dos sintomas ansiosos, após um único episódio de exercício com duração de algumas horas e até um dia após o exercício. Além disso, tem sido demonstrado que sessões agudas de atividade física promovem uma melhoria no estado de humor, como a diminuição de tensão/ansiedade, depressão e raiva e aumento do vigor, que podem durar horas após o exercício físico e que a repetição destes efeitos em longo prazo traria efeitos positivos para a saúde ${ }^{61-64}$.

Ainda, de acordo com Morgan (1985) ${ }^{65}$, North et al. (1990) ${ }^{66}$ e Ransford (1982)67, várias hipóteses psicológicas têm sido levantadas para explicar os efeitos benéficos do exercício físico sobre a saúde mental. As principais são: distração, autoeficácia e interação social. A hipótese distração, segundo os autores, sugere que a distração de estímulos desagradáveis levaria a uma melhora do humor durante e após o exercício; a autoeficácia pode explicar-se, pois o exercício físico pode ser visto como uma atividade desafiadora, pois se engajar em uma atividade física regular poderia levar a uma melhora do humor e da autoconfiança; já a interação social explica-se pelas relações presentes nas atividades físicas, assim como o suporte mútuo que os indivíduos engajados nela dão uns aos outros, isto teria participação importante nos efeitos do exercício sobre a saúde mental. Além disso, o efeito positivo do exercício físico sobre a saúde mental pode ser explicado por hipóteses fisiológicas, e entre elas, duas são mais estudadas: monoaminas e endorfinas. A primeira se apoia no fato de que a atividade física aumenta a transmissão sináptica das monoaminas, funcionando, supostamente, como os antidepressivos. A segunda hipótese, por sua vez, baseiase no fato de que a atividade física provoca a liberação de opioides endógenos (endogenous morphines), basicamente beta-endorfina. Supostamente os efeitos inibitórios dessas substâncias no sistema nervoso central seriam os responsáveis pela sensação de calma e melhora do humor que ocorre após o exercício físico ${ }^{65,67-70}$. No entanto, conforme Paluska e Schwenk (2000)71, ainda não há uma concordância quanto à importância relativa dessas hipóteses citadas (psicológicas e fisiológicas) para explicar a associação entre atividade física e melhora do humor, e um modelo psicobiológico que as combine parece ser o mais provável. Para uma definição precisa, seria necessário um conhecimento maior dos mecanismos que ligam à atividade física a cada uma dessas hipóteses e de mecanismos que as liguem à melhora do humor. De maneira suposta, com esse conhecimento, se chegará a um modelo em que fatores psicológicos e biológicos interajam de modo específico, variando segundo os estímulos ambientais e as características psicológicas e biológicas de cada indivíduo.

No grupo não praticante de exercício físico, os escores médios das escalas representadas pelos sentimentos negativos do POMS, ou seja, tensão, depressão, raiva, fadiga e confusão, aumentaram e a escala de vigor, sentimento positivo, teve uma pequena diminuição no decorrer das 12 semanas de estudo. O tipo de perfil iceberg, nesse grupo, não foi encontrado em nenhum período do estudo, evidenciando, desse modo, alterações negativas de humor. Essa diminuição da dimensão positiva, e o aumento das dimensões negativas, que ocorreram no decorrer do estudo, podem justificar-se em razão do sedentarismo e isolamento social; de acordo com Roeder (2003) $)^{30}$, o exercício físico canaliza as frustrações reprimidas e, quando praticado em grupo, promove a socialização, combatendo o isolamento social.

Porém, apesar dos resultados encontrados neste ensaio clínico confirmarem o que a literatura tem apontado ultimamente, o experimento apresentou algumas limitações: como grupo pequeno, devido ao período do ano (inverno) em que foi realizado o ensaio clínico, possível efeito placebo e possibilidade de melhor prognóstico das pacientes que participaram das sessões de hidroginástica. 


\section{CONCLUSÕES}

Portanto, um programa de exercícios físicos baseado em hidroginástica mostrou-se um auxiliar terapêutico benéfico no tratamento do transtorno de ansiedade, pois todas as pacientes do grupo de praticantes de exercícios físicos apresentaram redução do nível de ansiedade. Não houve eliminação completa da psicopatologia, porém os exercícios físicos influenciaram positivamente como complemento terapêutico no tratamento do transtorno de ansiedade quando comparado com o tratamento convencional apenas com medicamentos. Para o perfil de estado de humor, foram encontradas alterações no decorrer do estudo, no entanto apenas o grupo não praticante de exercícios físicos experimentou alteração negativa de humor durante o experimento, enquanto o grupo praticante de exercício físico evidenciou perfil de saúde mental positiva, demonstrando, também, o efeito agudo benéfico do exercício físico no decorrer do ensaio clínico.

\section{REFERÊNCIAS}

1. Andrade L, Lolio C, Gentil Filho V, Laurenti R. Epidemiologia dos transtornos mentais em uma área de captação da cidade de São Paulo, Brasil. Rev Psiq Clín. 2000;26:257-61.

2. Nunes Filho EP, Bueno JR, Nardi AE. Psiquiatria e saúde mental: conceitos clínicos e terapêuticos fundamentais. São Paulo: Atheneu; 2001.

3. Scarpato AT. 0 estranho que me habita: a síndrome do pânico numa perspectiva formativa. Rev Reich Instit Sed Sapientiae. 2001;10:50-66.

4. Margins R, Picon P, Cosner AF, Silveira R. Relação entre estressores, estresse e ansiedade. Rev Psiq Rio Grande do Sul. 2003;25(2):65-74.

5. Kinrys G, Wygant LE. Anxiety disorders in women: does gender matter to treatment? Rev Bras Psiquiatr. 2005;27(2):43-50.

6. Butler T, Pan H, Epstein J, Protopopescu X, Tuescher 0, Goldstein M, et al. Fear-related activity in subgenual anterior cingulate differs between men and women. Neuroreport. 2005;16(11):1233-6.

7. Pujol J, López A, Deus J, Cardoner N, Vallejo J, Capdevila A, et al. Anatomical variability of the anterior cingulate gyrus and basic dimensions of human personality. Neuroimage. 2002;15(4):847-55

8. Redmond G. Mood disorders in the female patient. Int Jour Fertil Wom Medicine. 1997; $42(2): 67-72$.

9. Shear MK. Anxiety disorders in women: gender-related modulation of neurobiology and behavior. Semin Reprod Endocrinology. 1997;15(1):69-76.

10. Altchiler L, Motta R. Effects of aerobic and nonaerobic exercise on anxiety, absenteeism, and job satisfaction. Jor Clin Psychiatry. 1994;50(6):829-39.

11. Broman-Fulks JJ, Berman ME, Rabian BA, Webster MJ. Effects of aerobic exercise on anxiety sensitivity. Beh Res Therapy. 2004;42(2):125-36.

12. Brown DR, Morgan WP, Raglin JS. Effects of exercise and rest on the state anxiety and blood pressure of physically challenged college students. J Sports Med Phys Fitness. 1993;33(3):300-5.

13. O'Connor PJ, Petruzzello SJ, Kubitz KA, Robinson TL. Anxiety responses to maximal exercise testing. Braz Jour Sport Medicine. 1995;29(2):97-102

14. Petruzzello SJ, Landers DM, Hatfield BD, Kubitz KA, Salazar W. A meta-analysis on the anxiety reducting effects of acute and chronic exercise. Outcomes and mechanisms. Sports Med. 1991;11(3):143-82.

15. Raglin JS, Turner PE, Eksten F. State anxiety and blood pressure following 30 min of leg ergometry or weight training. Med Sci Sports Exerc. 1993;25(9):1044-8.
16. Antunes HK, Stella SG, Santos RF, Bueno OFA, Mello MT. Depression, anxiety and quality of life scores in seniors after an endurance exercise program. Rev Bras Psiquiatr. 2005;27(4):266-71.

17. Melo MT, Bolosco RA, Esteves AM, Tufik S. 0 exercício físico e os aspectos psicobiológicos. Rev Bras Med Esporte. 2005;11(3):203-7.

18. Beck AT, Brown G, Epstein N, Steer RA. An inventory for measuring clinical anxiety. J Consult Clin Psychol. 1988;56:893-7.

19 Cunha JA. Manual da versão em português das Escalas de Beck. 1.ed. São Paulo: Casa do Psicólogo; 2001. p. 171.

20. Matsudo S, Araújo T, Matsudo V, Andrade D, Andrade E, Oliveira L, Braggion G. Questionário Internacional de Atividade Física (IPAQ): Estudo de Validade e Reprodutibilidade no Brasil. Rev Ativ Fís Saúde. 2001;6(2):5-18.

21. Borg GAV, Noble BJ. Perceived exertion. In: Wilmore JH (ed.). Exercise and sport sciences reviews. Vol. 2 New York: Academic Press; 1974. p. 131-53.

22. McNair D, Lorr M, Dopplerman L. POMS. San Diego, CA: Education and Testing Service; 1971.

23. Salmon P. Effects of physical exercise on anxiety, depression, and sensitivity to stress: a unifying theory. Clin Psychol Review. 2001;21(1):33-61.

24. Craft $L L$, Landers AM. The effects of exercise on clinical depression and depression resulting from mental illness: a meta-analyses. Jour Sport Exerc Psychology. 1998;20:339-57.

25. Gallo Jr L, Marin-Neto JA, Maciel BC, Golfetti R, Martins LEB, Chacon-Mikahil MPT, et al. Atividades físicas: "remédio" cientificamente comprovado? A Terceira Idade. 1995;10(6):34-43.

26. Goldfarb AH, Jamurtas AZ. Beta-endorphin response to exercise: an update. Sports Med. 1997;24:8-16.

27. Heitkamp HC, Huber W, Sheib K. Beta-endorphin and adrenocorticotrophin after incremental exercise and marathon running-female responses. Eur J Appl Physiol. 1996;72:417-24.

28. Brogan DR. Rehabilitation services needs: physician's perceptions and referrals. Arch Phys Med Rehabilitation. 1981;62:215

29. Cardoso JR. Atividades físicas para a terceira idade. A terceira idade. 1992;5(4):9-21.

30. Roeder MA. Atividade física, saúde mental e qualidade de vida. Rio de Janeiro: Afiliada; 2003.

31. Baldwin DS, Polkinghorn C. Evidence-based pharmacotherapy for generalized anxiety disorder. Int J Neuropsychopharm. 2005;8(2):293-302.

32. Eckerson J, Anderson T. Physiological response to water aerobics. J Sports Med Phys Fitness. 1992;32(3):255-61.

33. Kruel LFM. Alterações fisiológicas e biomecânicas em indivíduos praticando exercícios de hidroginástica dentro e fora d'água. Santa Maria. Tese de Doutorado. Universidade Federal de Santa Maria; 2000.

34. Vieira JLL, Porcu M, Rocha PGM. A prática de exercícios físicos regulares como terapia complementar ao tratamento de mulheres com depressão. J Bras Psiquiatr. 2007;56(1):23-8.

35. Alves RV, Mota J, Costa MC, Alves JGB. Physical fitness and elderly health effects of hydrogynastics. Rev Bras Med Esporte. 2004;10(1):38-43.

36. Avellini BA, Shapiro Y, Pandolf KB. Cardio-respiratory physical training in water and on Land. Eur J Appl Physiol. 1983;50:255-63.

37. Takeshima N, Rogers ME, Watanabe E, Brechue WF, Okada A, Yamada T, et al. Waterbased exercise improves health-related aspects of fitness in older women. Med Sci Sports Exerc. 2002;33(3):544-51.

38. Tauton JE, Rhodes EC, Wolski LA, Donelly M, Warren J, Elliot J, et al. Effect of land-based and water-based fitness programs on the cardiovascular fitness, strenght and flexibility of woman aged 65-75 years. Gerontology. 199642:204-10.

39. Rocha JCC. Hidroginástica: teoria e prática. Rio de Janeiro: Sprint; 1999.

40. Caromano FA, Themudo MR, Candeloro JM. Efeitos fisiológicos da imersão e do exercício na água. Fisioter Brasil. 2003;4(1)

41. Srámek P, Simecková M, Janski L, Savlíková J, Vybíral S. Human physiological responses to immersion into water of different temperatures. Eur J Appl Physiol. 2000;81:436-42.

42. Craig ABE, Dvorak M. Thermal regulation of man exercising during water immersion. Jour Appl Physiology. 1968;25:28-35.

43. Araújo CGS. Fisiologia do exercício físico e hipertensão arterial. Uma breve introdução. Rev Hipertensão. 2001:4(3):3-8. 
44. Rondon MUPB, Brum PC. Exercício físico como tratamento não farmacológico da hipertensão arterial. Rev Bras Hipertens. 2003;10:134-7.

45. Irigoyen MC, Angelis KD, Schaan BDA, Fiorino P, Michelini LC. Exercício físico no diabetes melito associado à hipertensão arterial sistêmica. Rev Bras Hipertens. 2003,10:109-17.

46. Silverthorn DU. Fisiologia integrada. Fisiologia humana. Uma abordagem integrada. 2.ed. Barueri: Manole; 2003.

47. Antunes HKM, Andersen ML, Tufik S, Mello MT. 0 estresse físico e a dependência de exercício físico. Rev Bras Med Esporte. 2006;12(5):234-8.

48. Werneck FZ, Bara Filho MG, Ribeiro LCS. Efeitos de exercício físico sobre os estados de humor: uma revisão. Rev Bras Psicol Esp Exercício. 2006;0:22-54.

49. Berger BG. Psychological benefits of an active lifestyle: What we know and what we need to know. Quest, Champaign, Illinois. 1996;1(48):330-53.

50. Berger BG, Mcinman A. Exercise and the quality of life. In: Singer RN, Murphey M, Tennant LK (eds.). Handbook of research on sport psychology. New York: Macmillan; 1993. p. 729-60.

51. Boutcher SH. Emotions and aerobic exercise. In: Singer RN, Murphey M, Tennant LK (eds.). Handbook of research on sport psychology. New York: Macmillan; 1993. p. 799-814.

52. American College of Sports Medicine. ACSM position stand: the recommended quantity and quality of exercise for developing and maintaining cardiorespiratory and muscular fitness, and flexibility in adults. Med Sci Sports Exerc. 1998;30(6):975-91.

53. Brown $R$, Harrison J. The effects of a strength training program on the strength and self concept of two female age groups. Res Q Exerc Sport. 1986;1(57):315-20.

54. Kesaniemi YK, Danforth EJr, Jensen MD, Kopelman PG, Lefêbure, P, Reeder BA. Dose-response issues concerning physical activity and health: an evidence-based symposium. Med Sci Sports Exerc. 2001;33(6):351-8.

55. Leon AS, Connett J, Jacobs Jr DR, Rauramaa R. Leisure-time physical activity levels and risk of coronary heart disease and death. The Multiple Risk Factor Intervention Trial. JAMA. 1987;258(17):2388-95.

56. Pilling S, Bebbington P, Kuipers E, Garety J, Orbach G, Morgan C. Psychological treatments in schizophrenia: meta-analysis of family intervention and cognitive behaviour therapy. Psychol Med. 2002;32:763-82.
57. Shephard RJ. Absolute versus relative intensity of physical activity in a dose-response context. Med Sci Sports Exerc. 2001;33(6):400-18.

58. Biddle S. Exercise and psychosocial health. Res Q Exerc Sport. 1995;66(4):292-7.

59. Raglin JS. Exercise and mental health - Beneficial and detriment effects. Sports Med. 1990;9(6):323-9.

60. Raglin JS, Morgan WP. Influence of exercise and quiet rest on states anxiety and blood pressure. Med Sci Sports Exerc. 1987;19:456-83.

61. Berger BG, Molt RW. Exercise and mood: a selective review and synthesis of research employing the profile of mood states. J Appl Physiol. 2000;12:69-92.

62. Dunn AL, Trivedi MH, O'neal HA. Physical activity dose-response effects on outcomes of depression and anxiety. Med Sci Sports Exerc. 2001;33(6):587-97.

63. Lane AM, Lovejoy DJ. The effects of exercise on mood changes: the moderating effect of depressed mood. J Sports Med Phys Fitness. 2001;41:539-45.

64. Toskovic NN. Alterations in selected measures of mood with a single bout of dynamic Taekwond exercise in college-age students. Percep Mot Skills. 2001;92(3):1031-8.

65. Morgan WP. Affective beneficence of vigorous physical activity. Med Sci Sports Exerc. 1985;17:94-100.

66. North TC, Mccullagh P, Tran ZV. Effect of exercise on depression. Exerc Sport Sci Rev. 1990;18:379-415.

67. Ransford (P. A role for amines in the antidepressant effect of exercise: a review. Med Sci Sports Exerc. 1982;4(1):1-10.

68. Allen M. The psychobiology of athletic training. In: Begel D, Burton RW (ed.). Sport Psychiatric: theory and practice. New York: W. W. Norton e Company; 2000. p. 22-44.

69. Dunn AL, Dishman RK. Exercise and the neurobiology of depression. Exerc Sport Sci Rev. 1991;19:41-98.

70. Nicoloff G, Schwenk TS. Using exercise to ward off depression. Phys Sport Med. 1995;23(9):44-58.

71. Paluska SA, Schwenk TL. Physical activity and mental health: current concepts. Sports Med. 2000;29(3):167-80. 